\title{
Arbor
}

\section{Participacion del Ejercito del Aire en misiones de apoyo a la sociedad}

\section{José Antonio Beltrán Doña}

Arbor CLXXI, 674 (Febrero 2002), 327-340 pp.

El autor en este artículo expone de forma amena, libre de tecnicismos, las misiones de apoyo del Ejército del Aire a la sociedad a la que sirve. En él se nos habla de temas tan sugerentes como:

- El apoyo a los Cuerpos y Fuerzas de Seguridad del Estado en su lucha contra la inmigración ilegal, el narcotráfico, la seguridad ciudadana...

- El apoyo al desarrollo científico y tecnológico, en el que se destaca la labor esencial del Centro Cartográfico y Fotográfico del Ejército del Aire para la confección de toda clase de cartografía, realización de control de plagas, etc. También se trata la labor del Centro de Medicina e Instrucción Aeroespacial en su faceta de estudio sobre los problemas fisiológicos en el área aeroespacial.

- El Servicio de Búsqueda y Salvamento (SAR) que con sus tres Escuadrones, los de Madrid, Canarias y Palma de Mallorca, cubre todo el territorio nacional.

- Además, entre otros muchos, también se citan con detalle los apoyos por el transporte, la asistencia en emergencias y catástrofes, evacuaciones de ciudadanos, etc.

\section{Introducción}

Todas las misiones de las Fuerzas Armadas se derivan de las obligaciones constitucionales o de los convenios y acuerdos internacionales que, al ser suscritos por España, forman parte de nuestro ordenamiento le- 
gislativo y se realizan en beneficio de la sociedad y en función de lo que ésta demanda. Como consecuencia las Fuerzas Armadas vienen desarrollando, desde hace mucho tiempo una serie de misiones en apoyo directo de la sociedad basadas en sus capacidades tecnológicas, operativas y logísticas, que se han puesto de manifiesto en diversas ocasiones tales como accidentes, catástrofes, etc, ocurridas, tanto en territorio nacional como en el exterior, en las que, bien por verse desbordados los medios disponibles de las Autoridades civiles responsables, o bien por ser las Fuerzas Armadas las únicas que disponían de las capacidades apropiadas, se hizo preciso recurrir a la colaboración de Fuerzas Armadas.

Por otra parte, en épocas recientes, la pertenencia a la Organización de Naciones Unidas, a la Alianza Atlántica y otras organizaciones internacionales, ha traído con ello una amplia participación en misiones internacionales de Apoyo a la Paz y de Ayuda Humanitaria, participación que ha sido muy bien acogida y valorada por la sociedad española.

A su vez, la nueva estrategia de la OTAN para el siglo XXI, aprobada en la primavera de 1999, plantea una revisión de los conceptos tradicionales, que aconseja abordar en profundidad cuales son las misiones que la sociedad demanda a sus Fuerzas Armadas.

En el nuevo marco estratégico, con la globalización, cambio de valores, modernidad, etc, el futuro de nuestras Fuerzas Armadas se encuentra sometido a un debate abierto por el propio Ministro de Defensa, que ha llevado a acometer una Revisión Estratégica, actualmente en curso. Esta Revisión Estratégica contemplará las nuevas misiones de las Fuerzas Armadas, no solamente en el nuevo contexto internacional sino en el futuro próximo, con una visión a medio plazo estimada de 15 años.

Por último, a la vista de lo sucedido el 11 de septiembre en Estados Unidos, será necesario analizar el nuevo papel de las Fuerzas Armadas, en misiones en el interior. La intervención, aunque fuese tardía, del sistema de Defensa Aérea de los EE.UU. ante un atentado criminal como el sucedido obligará a nuevos planteamientos de seguridad interior, en el que se resaltará sin duda la misión de Policía Aérea.

Por lo tanto, el Ejército del Aire como componente de las Fuerzas Armadas no permanecerá ajeno a esa nueva concepción estratégica que se derive de los trabajos en curso. En ella, quizás, como un subgrupo de misiones derivadas de la misión genérica de las Fuerzas Armadas que señala el Art. 8 de la Constitución, se contemplarán las misiones que las Fuerzas Armadas vienen ya realizando en beneficio de la sociedad y aquellas otras de nuevo cuño que resulten de la Revisión.

Las misiones en apoyo de la sociedad, se incardinan, principalmente, en la colaboración entre Autoridades Militares y Autoridades Civiles. 
Participación del Ejército del Aire en misiones...

Para el Ejército del Aire, como hemos citado, ello no supone ninguna novedad. La aviación de transporte ha estado presente en prácticamente todo el mundo, los aviones apagafuegos, el transporte de órganos, etc, son claros ejemplos de lo que el Ejército del Aire puede hacer, en beneficio directo de la sociedad a la que pertenece, y en lo que se profundizará a lo largo de este artículo.

\section{Legislación actual}

Se pueden considerar tres etapas dentro de lo que la legislación española entiende como legislación de emergencia: la preconstitucional, la promulgación de la Constitución Española de 1978 y posteriormente la etapa postconstitucional.

Sin ser exhaustivos en detallar toda la legislación de emergencia de la que se dispone en el ámbito nacional, parece oportuno citar aquella que tiene una implicación más directa con las Fuerzas Armadas en el ámbito que tratamos. Así tenemos, entre otras, la Ley 2/1975 de Protección Civil y el Decreto 1125/1976 de colaboración de las autoridades militares con las gubernativas. Esta normativa, en general, trata distintos aspectos conceptuales que podríamos sintetizar en:

* El entorno de colaboración de las FAS con las autoridades civiles

* El ámbito de la protección a la población civil y

* El campo de la defensa propiamente dicho

Conviene distinguir perfectamente lo que supone la aportación de recursos militares a los planes especiales para la protección de la población, cuya responsabilidad y elaboración recae en el Ministro del Interior (Dirección General de Protección Civil), de la posible aportación de recursos militares y civiles a una emergencia de carácter bélico.

Esta diferenciación se representa, a continuación, en un gráfico donde quedan plasmados estos aspectos.

Dicho ésto nos centraremos en la colaboración Fuerzas Armadas -Sociedad, en una emergencia de carácter NO BÉLICO.

\section{El Ejército del Aire en la sociedad}

Dentro de los estudios que se están llevando a cabo con motivo de la Revisión Estratégica de la Defensa, en el apartado misiones se contemplará como pueden contribuir las Fuerzas Armadas con otras instituciones del Estado para preservar la seguridad y el bienestar de los ciudadanos. 


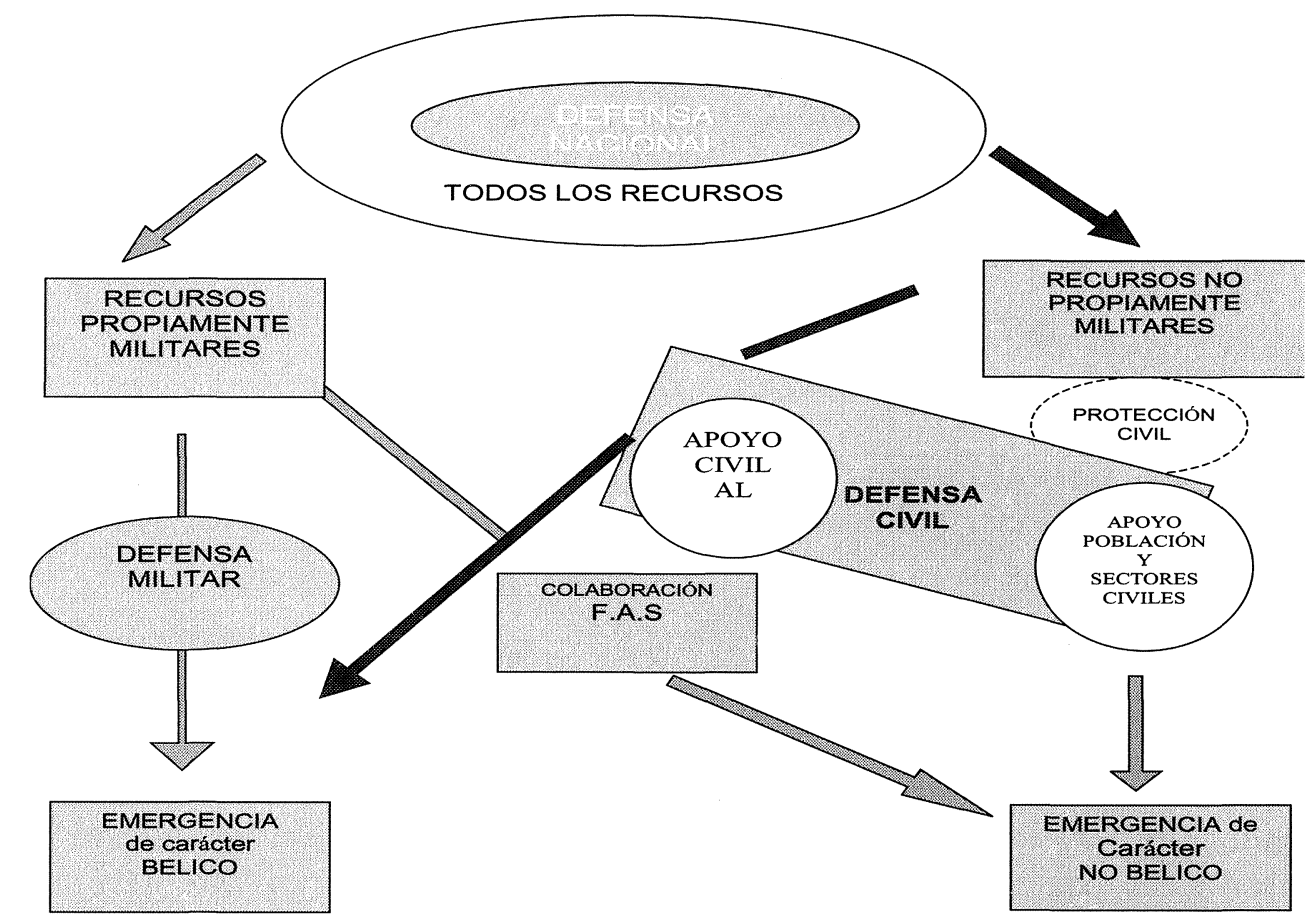

Sin que el catálogo que se cita a continuación sea exclusivo, entre las misiones en las que el Ejército del Aire puede colaborar en el marco general de las Fuerzas Armadas se pueden contemplar las siguientes:

- Apoyo a los Cuerpos y Fuerzas de Seguridad del Estado

- Apoyo al desarrollo científico y tecnológico

- Apoyo por el transporte

- La lucha contra incendios

- Vigilancia aduanera

- La búsqueda y el salvamento

- Apoyo a la exportación

- Asistencia en emergencia y catástrofes

- Evacuación de ciudadanos

- Etc.

\section{Apoyo a los Cuerpos y Fuerzas de Seguridad del Estado}

Los medios aéreos de los que actualmente está dotado el Ejército del Aire disponen de capacidades para apoyar por ejemplo, la lucha contra la inmigración ilegal, el narcotráfico, la seguridad ciudadana, etc. 
Participación del Ejército del Aire en misiones...

Sin entrar en detalles sobre los medios tecnológicos sí citaremos algunas de estas colaboraciones que, de hecho y de acuerdo con las capacidades actuales, se vienen prestando.

Los rádares pueden tener cierta capacidad de detección a gran distancia y permiten, en algunos casos y si así se programa, el poder hacer seguimientos de buques, y otros tipos de embarcaciones que se puedan dedicar al tráfico ilícito, además de la seguridad que suponen para el tráfico aéreo civil. Quizás, éste es un aspecto poco conocido por la opinión pública: la señal que los rádares militares captan es transferida a la aviación civil para el control de la circulación aérea general. Extractores de datos establecidos en determinados rádares facilitan esta labor que llevan a cabo las agencias de control de tráfico aéreo, en tiempo de paz.

Por otra parte, las necesidades urgentes de despliegue de las Fuerzas de Seguridad del Estado son atendidas, en ocasiones, por los aviones de transporte. Su disponibilidad, con aviones en alerta en todas las unidades de transporte, Zaragoza, Getafe y Valladolid los hace sumamente útiles ante circunstancias sobrevenidas.

Contingentes policiales han sido trasladados a distintos puntos de la geografía nacional por aviones del Ejército del Aire. Traslado de presos, de funcionarios del Estado que por necesidad urgente han tenido que asistir a reuniones y conferencias, etc, son misiones habituales.

Los aviones de Patrulla Marítima, en sus misiones de vigilancia de los espacios marítimos de interés y responsabilidad española han contribuido a la detección y seguimiento de embarcaciones dedicadas al narcotráfico. La colaboración con las autoridades civiles responsables ha permitido en muchas ocasiones la detención de embarcaciones que transportaban importantes alijos.

En el mes de diciembre de 2001, se firmó un convenio de colaboración entre los Ministerios de Interior y de Defensa, que permite regular la colaboración y cooperación entre ambos, estableciendo puntos de enlace, procedimientos de petición de medios, etc..

\section{Apoyo al desarrollo científico y tecnológico}

Muchos son los campos en los que el Ejército del Aire colabora en este ámbito tan importante para el desarrollo nacional. Por citar algunos tenemos la producción de fotografía y de cartografía aeronáutica, estudios en el ámbito de la medicina aerospacial y el apoyo a la exportación de la industria española de defensa. 
El Centro Cartográfico y Fotográfico del Ejército del Aire, de Cuatro Vientos, además de los trabajos propios que realiza en beneficio del Ejército del Aire como son la confección de la cartografía aeronáutica militar de España, fichas de penetración en bases y aeródromos militares, así como de las instalaciones abiertas al tráfico civil, para lo que dispone de los medios más avanzados existentes hoy en el mercado, presta apoyo a vuelos científicos de control de polución, de plagas, etc.; realiza levantamientos cartográficos para resolución de conflictos en beneficio del Ministerio de Justicia. Es, además, el responsable de la censura de los vuelos fotográficos sobre áreas prohibidas o restringidas; correspondiéndole en estas áreas la elaboración de los elementos fotográficos necesarios para la ejecución de los planes de urbanismo.

En el área de la enseñanza y la investigación tiene convenios de colaboración con distintas Universidades entre otras, con la Complutense (Geografía e Historia e Ingenieros Topógrafos), con la de Oviedo, etc.

Dispone de un archivo fotográfico de toda la geografía nacional abierto a todos los ciudadanos españoles.

No son éstos todos los cometidos que el Centro Fotográfico y Cartográfico del Ejército del Aire desarrolla en beneficio del resto de la sociedad española, pero sí un resumen de los más importantes.

En el campo de la medicina es de reseñar la importantísima labor que desarrolla el Centro de Instrucción de Medicina Aerospacial (CIMA).

El Centro de Instrucción de Medicina Aerospacial, CIMA, siglas reconocidas y prestigiadas en todo el ámbito aeronáutico nacional e internacional, lleva años dedicado al campo de la medicina aerospacial. Sería muy prolijo detallar todas las tareas que realiza, por lo que únicamente se hará un breve resumen de ellas.

$\mathrm{Su}$ actividad la podemos considerar dividida en tres grandes sectores: reconocimientos médicos y entrenamiento fisiológico, la enseñanza, la formación y la investigación y sus colaboraciones científicas.

En el apartado de reconocimientos médicos es, junto con la Clínica Quirón de Barcelona, el único centro autorizado por la Dirección General de Aviación Civil para todos los reconocimientos iniciales para la Aviación General, pilotos, pilotos privados, ingenieros de pruebas, controladores, etc.

En cuanto al entrenamiento fisiológico, ha apoyado y realizado estudios sobre problemas fisiológicos relacionados con el medio hipobárico en expediciones de alta montaña. En concreto colaboró con el equipo de TVE «Al filo de lo imposible», con un equipo de alta montaña de la ONCE, con el departamento de Medicina del Deporte de la Complutense y con la expedición al Himalaya de Castilla y León. 
En el ámbito de la enseñanza, la formación y la instrucción, su personal médico especialista, altamente cualificado, figura como miembro de la Comisión Nacional de la Especialidad (Presidente, secretario y vocal), ocupan cargos representativos en la Sociedad Española de Medicina Aerospacial, Academia Internacional de Medicina de Aviación y del Espacio, Sociedad Americana de Medicina Aerospacial y Asociación Iberoamericana de Medicina de la Aviación y del Espacio.

En el campo del apoyo al tráfico aéreo civil, destaca la Calibración de ayudas.

\section{La Calibración de Ayudas.}

Se ha comentado en un párrafo anterior, la aportación de la señal de los rádares militares para la seguridad de la navegación aérea general. Esta Seguridad en Vuelo, se completa en tierra con la comprobación y certificación del funcionamiento correcto de las ayudas a la navegación, más conocido como calibración, nombre que recibió en sus inicios el 45 Grupo de Fuerzas Aéreas.

Aviones del Ejército del Aire de esta Unidad verifican, desde el año 1956, que determinadas ayudas a la navegación en tierra, las cuales permiten a los aviones realizar sus vuelos con seguridad, funcionan dentro de los parámetros establecidos. Para ello la unidad dispone de aviones especialmente preparados con equipos de altísima fiabilidad que permiten comprobar un número muy elevado de ayudas.

\section{La ayuda humanitaria. Apoyo por el transporte}

¡Cuántas veces se ha visto un avión del Ejército del Aire en misiones de Ayuda humanitaria despegando a cualquier hora del día o de la noche con destinos tan lejanos como Perú, Mozambique, etc.!

Hemos citado anteriormente la alta disponibilidad de las tripulaciones y los medios del Ejército del Aire y vuelve a ser, en función de ella, la causa principal que motiva su participación en misiones como las citadas. Esta disponibilidad llega a tal punto que, en algunos casos, ante una solicitud de las organizaciones de ayuda humanitaria para transportar una carga al lugar más alejado del mundo ha estado el avión situado y preparado en la correspondiente base, antes de que lo estuviese la propia carga a transportar. Vuelos de una duración superior a 16 horas, han sido ejecutados con magnífica puntualidad y perfección acudiendo con la ayuda en socorro de países amigos, incluso con riesgo para 
la propia aeronave y su tripulación por tratarse de países en conflicto. Evacuaciones de ciudadanos en situaciones casi extremas, sobrevuelo de países y territorios en conflicto, escalas técnicas necesarias en aeropuertos de países con escasa infraestructura, son condiciones normales de operación. Pero ahí han estado las unidades de transporte cumpliendo y ejecutando, como es su obligación, las misiones que les ha asignado el Gobierno.

El ya citado $\mathbf{4 5}$ Grupo de Fuerzas Aéreas, cuando hablábamos de calibración, compagina estos cometidos con los del traslado de autoridades. Sus aviones y tripulaciones han viajado por los cinco continentes y han transportado a numerosos dirigentes nacionales y de otros estados.

No son éstas las únicas misiones que realiza esta pequeña, pero importantísima unidad. Además de las específicamente militares como las de reabastecimiento en vuelo, hay que contabilizar las de transporte de personal, la repatriación de españoles y otras nacionalidades donde zonas de conflicto y la ayuda humanitaria que son otras de las tareas a las que se dedica y ha dedicado un importante esfuerzo.

Argelia, Angola, Abudhabi son algunos de los lugares desde los que se han repatriado españoles.

En cuanto a la ayuda humanitaria, normalmente en estrecha relación con la Agencia Española de Cooperación Internacional, se ha transportado ayuda, con ocasión de catástrofes: terremotos, inundaciones, hambrunas. El Salvador, Perú, Mozambique, etc, y en especial toda Centroamérica, son lugares en los que, desde los primeros instantes de la tragedia ha estado presente la Fuerza Aérea Española.

La bases aéreas de Zaragoza, donde están estacionados los aviones C130 Hércules, los «Dumbo» y la de Torrejón colaboran con sus instalaciones y apoyan la mayor parte de las operaciones de ayuda humanitaria que se realizan. En la citada base de Torrejón se dispone de hangares para guardar la ayuda que aportan las organizaciones gubernamentales y no gubernamentales hasta su carga en los aviones.

Los profesionales de la unidad trabajan horas y horas para disponerla en las plataformas adecuadas para su carga, estiba y posterior descarga con las condiciones de seguridad necesarias para el vuelo. La carga se produce a veces a altas horas de la noche, durante horas, pero finalmente con puntualidad estricta el avión despega hacia su destino.

Para ello, supervisores de carga, soldados de las unidades de apoyo y los servicios de nuestras bases aéreas han estado trabajando duramente, sin descanso, durante muchas horas para que se cumpliera con éxito la misión, en ese momento, encomendada. 


\section{La lucha contraincendios}

El Ministerio de Defensa y el Ministerio de Medio Ambiente han regulado su cooperación en el ámbito de la extinción de incendios forestales mediante la firma de un convenio por el cual el Ejército del Aire ha estado operando durante muchos años los aviones CL-215 T, Canadair, más conocidos como «apagafuegos».

El 43 Grupo de Fuerzas Aéreas, ubicado en la Base Aérea de Torrejón es el responsable de la operación de los medios aéreos de ICONA.

Durante años sus aviones con los colores rojo y amarillo se han dejado ver en prácticamente todos los incendios que han asolado durante los veranos nuestra geografía.

Un invierno trabajando y progresando para durante el verano rendir con la máxima disponibilidad y eficacia en la lucha contra incendios. Con tomas de agua en lugares difíciles, pantanos, desembocaduras de ríos, bahías, etc. distribuidos por toda la geografía, Mallorca, Málaga, etc, se ha visto operar estos aviones. Tripulaciones que han arriesgado sus vidas, hasta en algunos casos perderlas por salvar las de los demás, jalonan la vida de esta unidad.

Cuando llega el verano despliegan por todo el territorio y establecen un servicio de máxima disponibilidad desde que sale el sol hasta que se pone.

Volar entre el humo, con altas temperaturas, turbulencias, visibilidad reducida, rodeados de montañas agrestes y con difícil acceso son los servicios habituales de estas tripulaciones.

\section{La Vigilancia Aduanera}

Con anterioridad al año 1996 la labor correspondiente a la operación de los medios aéreos de Vigilancia Aduanera (VA). estaba encomendada a una empresa civil. Durante el primer semestre del año 1996 entre el Ministerio de Economía y Hacienda (Agencia Estatal de Administración Tributaría, A.E.A.T.) y el Ejército del Aire (E.A.), se establecieron contactos que condujeron a la elaboración de un Convenio de Cooperación entre las citadas partes, que aseguraba las prestaciones necesarias por parte del E.A. en lo concerniente a la operación técnica de vuelo de los aviones y al mantenimiento de los mismos hasta el segundo escalón.

La misión fue asumida por el Ala 3 7, Villanubla, Valladolid. 
Para el Ejército del Aire suponía un nuevo reto la realización de las misiones de V.A., aunque el hecho de contar con la experiencia adquirida anteriormente, al haber organizado destacamentos como el que prestaba apoyo a la Misión Militar Española de Cooperación, con el Gobierno de la República de Guinea Ecuatorial, y como el que se realiza en Vicenza (Italia), fue de gran ayuda para la preparación de los destacamentos, tanto en el aspecto de personal como del material que sería preciso desplazar a los puntos de despliegue.

El año 1996 se produjo la activación inmediata de los Destacamentos, dándose un plazo de 24 horas para que los aviones desplegados pudieran ser operados en misiones de Vigilancia Aduanera (V.A). Ese mismo día se iniciaba el despliegue de los destacamentos. Los medios personales y materiales ya estaban preparados y se estaba a la espera de la orden por lo que de inmediato se desplazaron a los puntos previamente designados, las tripulaciones y aviones operativos para comenzar las misiones.

El fin principal de las misiones aéreas de V.A., es efectuar la vigilancia y reconocimiento marítimo para buscar, localizar, identificar, seguir a embarcaciones y conducir hacia ellas a otros medios, al objeto de investigación, represión y persecución de delitos de contrabando.

De todas las funciones asignadas a V.A. cabe destacar las siguientes:

* Dirección y coordinación de la lucha contra el fraude aduanero en general.

* Persecución, descubrimiento y represión en todo el Territorio Nacional, aguas jurisdiccionales y espacio aéreo español de los actos de contrabando de especies amenazadas o en peligro de extinción, mercancías prohibidas (marcas falsas, por ejemplo), etc., y en especial de los delitos de tráfico de drogas.

Hasta finales del mes de septiembre de 2001 se han realizado algo más de 18.000 H.V.. Hasta el año 1997 las horas eran realizadas exclusivamente con los aviones de la flota del Ala 37. A partir de 1998 en que empezaron a operar los aviones de la serie 200 procedentes de V.A., la carga de trabajo fue repartiéndose, aunque en la actualidad la operatividad requerida de los destacamentos no se podría mantener sin la concurrencia de los T-12B del Ala 37.

Como resumen final se puede decir que las misiones pueden variar desde el tedio producido por cuatro a cinco horas en línea de vuelo sin observar nada significativo y volver a casa, a estar continuamente bajando a dar pasadas de reconocimiento y observar como se arrojan bultos al mar precipitadamente, o como llega un barco de V.A. o Guardia Civil a interceptar a aquél que hemos estado siguiendo durante largas horas, incluso en distintos vuelos a lo largo de días. 


\section{La Búsqueda y el Salvamento}

El SAR, Servicio Aéreo de Rescate, traducción muy libre y poco exacta del término inglés SEARCH AND RESCUE (SAR), Búsqueda y Salvamento, es un conjunto de centros y unidades que tienen asignada internacionalmente un área de responsabilidad para la búsqueda y el rescate en caso de accidente aéreo. Su despliegue cubre todo el territorio nacional.

La perfecta conjunción de medios, Centros de Control de Salvamento, aviones y helicópteros facilita la compleja operación de la alerta, la búsqueda y posteriormente el rescate y salvamento.

Sin embargo, en multitud de ocasiones, estos medios, especialmente los helicópteros, han participado en el salvamento de vidas humanas originados por motivos distintos a los de su verdadera atención, el derivado de accidentes aéreos.

El salvamento de vidas en la mar, ha sido una constante en la larga historia del SAR. El caso del CASON, aquel buque que encalló, y se partió en dos, frente a las costas gallegas, donde un helicóptero y sus rescatadores salvaron un gran número de tripulantes en condiciones meteorológicas adversas.

Pescadores afectados de enfermedades que requerían evacuación inmediata, náufragos, etc, pueden testimoniar la labor del SAR. Su actividad es constante y su nivel de entrenamiento muy alto.

Cuantas veces en la bahía de Pollensa se ha visto a sus helicópteros realizar ejercicios de adiestramiento, recoger náufragos simulados, localizar un pequeño flotador en la inmensidad del mar. Especialmente resulta muy espectacular la transferencia de un rescatador, desde un helicóptero a una lancha en navegación y su posterior recuperación con una persona colgada de un atalaje especial o perfectamente izado con una camilla, etc.

Para todos ellos el salvamento de una sola vida es suficiente compensación a sus esfuerzos, a sus horas de alerta, a sus duras y largas horas de vuelos de búsqueda, etc.

\section{Apoyo a la exportación}

El Ejército del Aire está presente en prácticamente todas las demostraciones aeronáuticas que se realizan en el mundo, como mínimo en las más importantes, donde muestra y demuestra la calidad de los productos aeronáuticas nacionales. En muchos casos coincide con CASA, actual EADS, y en otros apoya con aviones o tripulaciones. 
Además, basándose en convenios bilaterales, ha prestado asistencia e incluso medios, en beneficio de la industria nacional para operar en otros países. En Suiza, por ejemplo, estuvo operando durante varios meses un avión CN-235, como apoyo subsidiario a un acuerdo de CASA. Estas y otras aportaciones han supuesto, en cualquier caso, que determinados países se inclinaran por la adquisición de medios de transporte aéreo de producción nacional.

Esto supone para el Ejército del Aire un notable esfuerzo, pero lo ve magníficamente recompensado, si de él se deriva un beneficio para nuestras empresas y por consiguiente para una buena parte del conjunto de la sociedad, a la que pertenece.

\section{La Cultura}

Hace tiempo leía un artículo que se iniciaba de la siguiente forma:

A los que han hecho la historia

A los que la investigan

A los que la reconstituyen

A los que la custodian

A los que la muestran

A los que la hacen y la harán

El citado artículo estaba dedicado a exponer que es, que hace, donde está, etc, el Servicio Histórico y Cultural del Ejército del Aire. Tiene como finalidad la investigación histórica, aeronáutica y espacial, la divulgación y difusión de los conocimientos aeronáuticos y astronáuticos y de la historia de la Aviación Española, destacando sus principales gestas.

Este Servicio, en su conjunto, es un esfuerzo más del Ejército del Aire en trasmitir a la sociedad su historia.

El Instituto de Historia y Cultura Aeronáutica es el órgano fundamental del Servicio en lo relativo a la investigación histórica, aeronáutica y espacial; la divulgación y difusión de los conocimientos aeronáuticas y de la historia de la Aviación Española. A él están adscritas la Revista de Aeronáutica y Astronáutica y la Revista Aeroplano.

Estas revistas, de divulgación aeronáutica, constituyen ese complemento esencial que permite al Ejército del Aire hacer llegar a todos los españoles, noticias históricas y otras de máxima actualidad.

La primera de ellas, la de Aeronáutica y Astronáutica, data de 1932, está dedicada a la actualidad y deja constancia en el futuro.

La segunda, la Revista Aeroplano, está más dedicada a difundir trabajos de investigación histórica. 
El Archivo Histórico del Ejército del Aire es el órgano encargado de reunir, conservar, ordenar y proporcionar documentos producidos por el Ejército del Aire en el ejercicio de sus actividades, al servicio de la investigación, la cultura, la información y la gestión administrativa.

No se puede dejar de recomendar un paseo, que puede ser familiar, con niños incluidos en una mañana madrileña, de esas que hay que consumir, visitando el Museo del Aire. A través de su recorrido podemos conocer la historia de la aviación, especialmente de la aviación española.

El Museo de Aeronáutica y Astronáutica es el órgano del Servicio Histórico y Cultural que, con carácter permanente, adquiere, conserva, investiga, comunica y exhibe, para fines de estudio, educación y contemplación, los fondos históricos, artísticos, científicos y técnicos, relacionados con la actividad aeronáutica para general conocimiento e ilustración de la Aeronáutica española y su desarrollo técnico, así como contribuye a la salvaguarda de sus tradiciones y difunde las glorias aeronáuticas españolas.

El Museo se instaló en Cuatro Vientos, cuna de la Aviación Militar española en el año 1975, en él se pueden contemplar, objetos, personajes, aviones, etc, que no son más que un pequeño recuerdo de la Historia de España, desde el punto de vista de la historia aeronáutica.

\section{Conclusión}

Las Fuerzas Armadas están al servicio de la Nación y, en consecuencia de la sociedad, y deben ser los ciudadanos los principales receptores de los beneficios de sus actuaciones.

Es comprensible que, normalmente, el hombre de la calle, el ciudadano de a pie en términos coloquiales, no perciba directamente los resultados. En primer lugar porque los beneficios normalmente recaen en el conjunto de la sociedad y en segundo lugar porque, desgraciadamente, en muchos casos el cumplimiento de estas misiones se hacen con el silencio callado del servicio cumplido, sin airear y publicar a los cuatro vientos HEMOS SIDO NOSOTROS.

A lo largo de este artículo, en el que estoy seguro se han quedado cosas en el tintero, donde las unidades que se citan no verán bien reflejada su dedicación y esfuerzo, se ha recorrido, prácticamente, toda la geografía nacional.

Con él, simplemente, se ha querido trasmitir lo que el Ejército del Aire viene realizando, año tras año y día tras día en apoyo directo de la sociedad. 
No sería correcto terminar sin una referencia a las Reales Ordenanzas para las Fuerzas Armadas definidas en su artículo uno como «el marco que define las obligaciones y derechos de sus miembros. Tienen por objeto preferente exigir y fomentar el exacto cumplimiento del deber inspirados en el amor a la Patria y en el honor, disciplina y valor». Estos son los grandes valores que hacen que los miembros del Ejército del Aire estén siempre dispuestos para cumplir, con la mayor eficacia, las misiones que el Gobierno les encomiende, en beneficio de la sociedad a la que pertenecen y, por lo tanto, al del pueblo español al que sirven. 